\title{
Internal motion prediction using kernel density estimation and general canonical correlation model
}

\author{
Alnowami, M.; Lewis, E.; Wells, K.
}

\begin{abstract}
This paper presents preliminary work in developing a global correlation model between lung tumor respiratory motion and external surrogate motion in external beam radiotherapy. This involves using a combination of set of dynamic CT datasets to train a bivariate kernel density estimation model. Canonical correlation analysis (CCA) is used to parametrize the correlation between the external observation surrogate and the target region, in this case tumor temporal motion. Such an approach is non-invasive and non-ionizing, and minimizes the patient setup time. Preliminary results shows that the correlation coefficient for preliminary data is high, ranging between 0.87 and 0.99 . Recasting the internal and external motion into eigenspace reveals the underlying correlation in an efficient and compact manner. A leave-one-out method was used to validate the proposed algorithm. The average error of tumor position was about $1.6 \mathrm{~mm}$.
\end{abstract}

This paper appears in: Nuclear Science Symposium and Medical Imaging Conference (NSS/MIC), 2011 IEEE, Issue Date: 23-29 Oct. 2011,

\section{INTRODUCTION}

One of the current major challenges in external beam radiotherapy for lung tumor is respiratory motion. There is a significant clinical evidence on the effect that respiratory motion has on lesions localization [1] ] [2]. Several studies to overcome tumor respiratory motion using different respiratory correlation models exist. These studies can be divided into two major methods; either directly by using an implanted fiducial marker in or near the tumor []ㅡ, []ㅡ or indirectly by using external thoracic/abdomen surface as a surrogate for the tumor motion []․ However using a fiducial marker in or near the lung tumor is limited due to increased risk of pneumothorax []], []]. Moreover, it is difficult to track the 3D position of the tumor using X-ray fluoroscopy alone without using an implemented marker. Furthermore, increasing the X-ray fluoroscopy dose or sampling rate is clinically problem-atic. Moreover, to overcome system latency due to image acquisition, data analysis, streaming, mechanical process and delivering, a prediction model is required.

In this work, the 3D tumor positions were predicted using 3D thoracic/abdomen surface motion as an external surrogate for internal tumor motion. This novel prediction model was built using a patient specific prediction model of respiration [8] and a generative correlation model. A set of eight simulated dynamic CT datasets and five lung cancer patients dynamic CT datasets were used to build the generative correlation model in this preliminary study. The internal tumor and external surrogate position was used to train the multivariate kernel density estimation to build the prediction model. Canonical correlation analysis (CCA) was then used to parametrize the correlation between the external surrogate and the actual temporal tumor motion. 


\section{METHODOLOGY AND PRELIMINARYRESULTS}

\section{A. Methodology}

The global model is built on our prior work presented at MIC2010 [8] by tracking the anterior surface temporal motion during a Dynamic CT scan using a marker-based tracking system, extracting the temporal variation and projecting the captured motion sequence data into a lower dimensional space using Principal Component Analysis (PCA). Although, in this latest study the external surface temporal motion was extracted from the dynamic CT data, the same method describe in [ 8 ] for modeling the external surface temporal motion was used in this study. The 3D lung tumor position was segmented from the captured dynamic CT data. The center of mass of the tumor position in each phase was extracted to represent the 3D displacement of the tumor during respiration. The temporal motion of the tumor and the external surface were correlated in the eigenspace using Canonical Correlation Analysis (CCA). A three dimensional matrix covers the likelihood of each position/phase configuration of the Thoracic surface/Abdomen surface, temporal motion of the tumor in the Eigenspace and the velocity of the motion from one position to the next will be used to train the multivariate kernel density estimation model to build the prediction and correlation model.

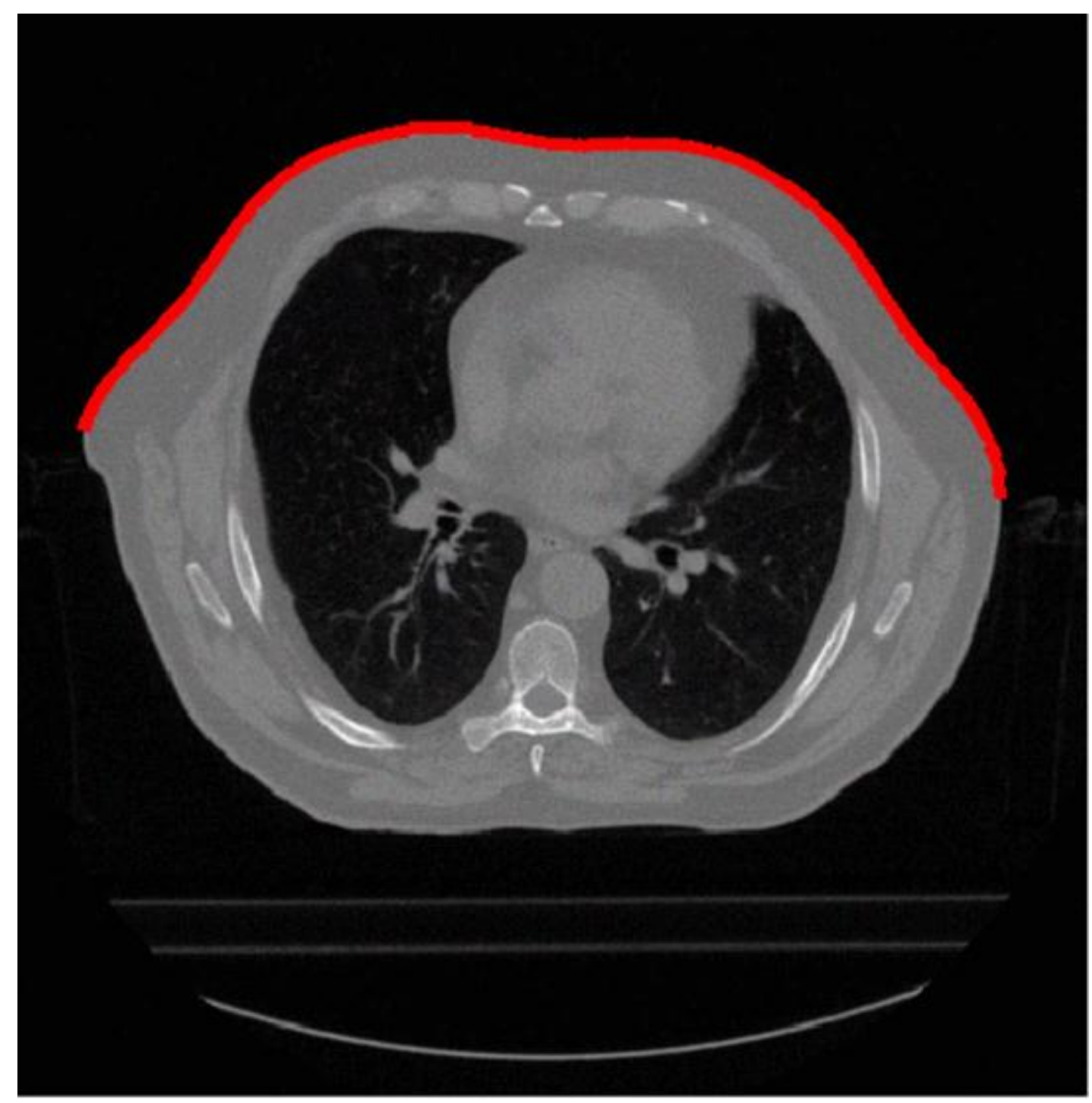

Fig. 1. An example of the surface detection method. An intensity threshold method with a canny edge detector was used to extract the upper skin surface. 


\section{B. External Surface temporal motion}

In this study the external surface temporal motion was extracted from the dynamic CT data. An intensity threshold method with an edge detector algorithm was used to extract the upper skin surface. Figure 1 shows a trans-axial slice with the detected surface.

For each subjects dataset the capture motion sequences during the dynamic CT scan will be arranged as a column matrix $F_{n}$ :

$$
F_{n}=\left[X_{n, 1}, Y_{n, 1}, Z_{n, 1}, \ldots \ldots \ldots \ldots \ldots \ldots, X_{n, s}, Y_{n, s}, Z_{n, s}\right]^{T}
$$

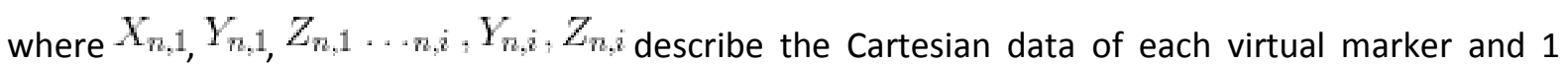
through $i$ is the marker index at frame $n$. As a result each frame will be represented by $D * 1$ vectors, where $D=3 * i$ is the dimensional vectors for $F_{n}$. The respiratory motion sequence for each volunteer is represented by a matrix $M=\left[F_{1}, \ldots \ldots \ldots, F_{t}, \mathrm{t}\right.$ is the number of respiratory phases, where each row of the motion matrix corresponds to all the motion components of a particular marker, and each column of $M$ corresponds to a set of measurements from one particular frame.

Principal component analysis (PCA) was used to reduce the dimensionality of the acquired data $M$ and analyze the most significant aspect of temporal variation without sacrificing significant accuracy [9]. The dimensionally reduced external surrogate data will be represented as a vector $p$.

\section{Tumor temporal motion}

The tumor position was segmented using ITK-SNAP [10] for each phase in CT dynamic data.Figure $\underline{2}$ and Figure 3 illustrates an example of the tumor, lung and trachea segmented volume for one phase of the respiratory cycle.

The center of mass $C$ for each tumor position was calculated and represented by a matrix $C_{n}=$ where $c$ is a $3 \mathrm{D}$ column vector represents the anterior-posterior (AP), lateral (L), and superior-inferior (SI) position of the center gravity of the tumor and $t$ is the number of phases extracted from the dynamic CT data. Figure 4 Illustrates the anterior-posterior (AP) (Z direction), lateral (L) (X direction), and superior-inferior (SI) (Y direction) temporal motion of the center gravity of the tumor.

\section{Canonical Correlation Analysis (CCA)}

CCA was used to measure the relationship between the external surrogate $P$ and the tumor center $C$. Canonical correlation analysis can be defined as the problem of finding two sets of basis vectors, one for the external surrogate motion and the other for the tumor temporal motion, such that the correlations between the projections of the variables onto these basis vectors are mutually maximized. Canonical correlation analysis seeks vectors $w_{P}$ and $w_{C}$ such that the random variables $u=w_{P}^{T} P$ and $v=w_{C}^{T} C$ maximize the correlation $\rho=\operatorname{cor}(u, v)$. The correlation coefficient $\rho$ is calculated by equation 2 . 


$$
\begin{aligned}
\rho & =\frac{E[u v]}{\sqrt{E\left[P^{2}\right] E\left[C^{2}\right]}} \\
& =\frac{E\left[w_{P}^{T} P C^{T} w_{C}\right]}{\sqrt{E\left[w_{P}^{T} P P^{T} w_{P}\right] E\left[w_{C}^{T} C C^{T} w_{C}\right]}} \\
& =\frac{w_{P}^{T} k_{P C} w_{C}}{\sqrt{w_{P}^{T} k_{P P} w_{P} w_{C}^{T} k_{C C} w_{C}}}
\end{aligned}
$$

where $k$ is the covariance matrix denoted either as the within-sets covariance matrices $k_{C C}$, or the between-sets covariance matrices as $k_{P C}$. The maximum canonical correlation is the maximum of $\rho$ with respect to $w_{C}$ and $w_{P}$. In this preliminary study, the absolute value of the correlation coefficient $\rho$ was ranging between 0.87 and 0.99 . This values indicates a high correlation between the external surrogate and tumor respiratory motion. Figure 6 illustrates the external surrogate phases verses the tumor phases in the Eigenspace. Figure 7 illustrates the total data used to train the kernel, where the red line indicates the external surrogate and the blue line indicate the tumor motion in the Eigenspace. Thus, the aforementioned Recasting of the internal and external motion into Eigenspace reveals the underlying correlation between them.

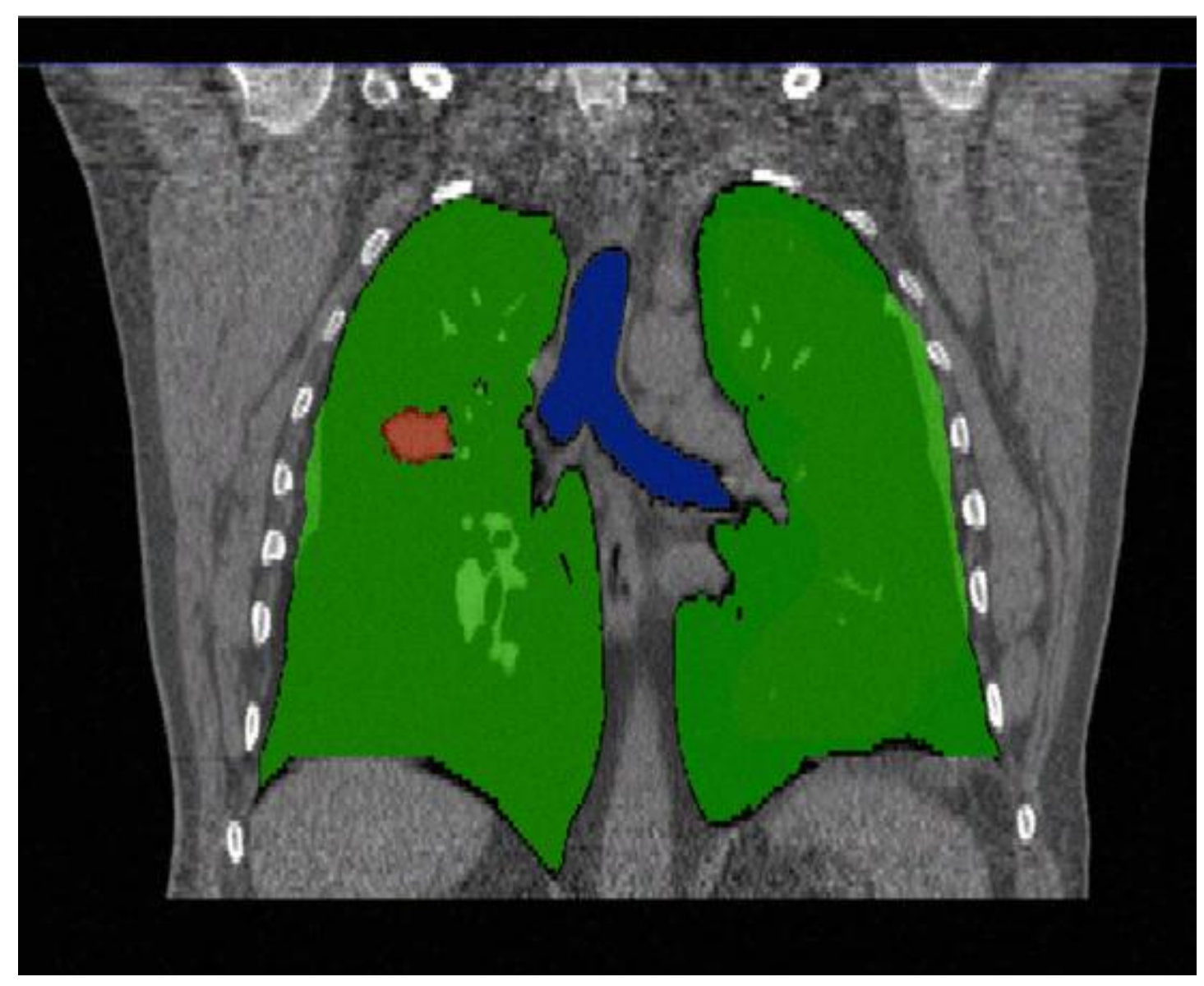

Fig. 2. An example of the segmented tumor position for one phase using ITK-SNAP. The red marked area indicates the tumor. 


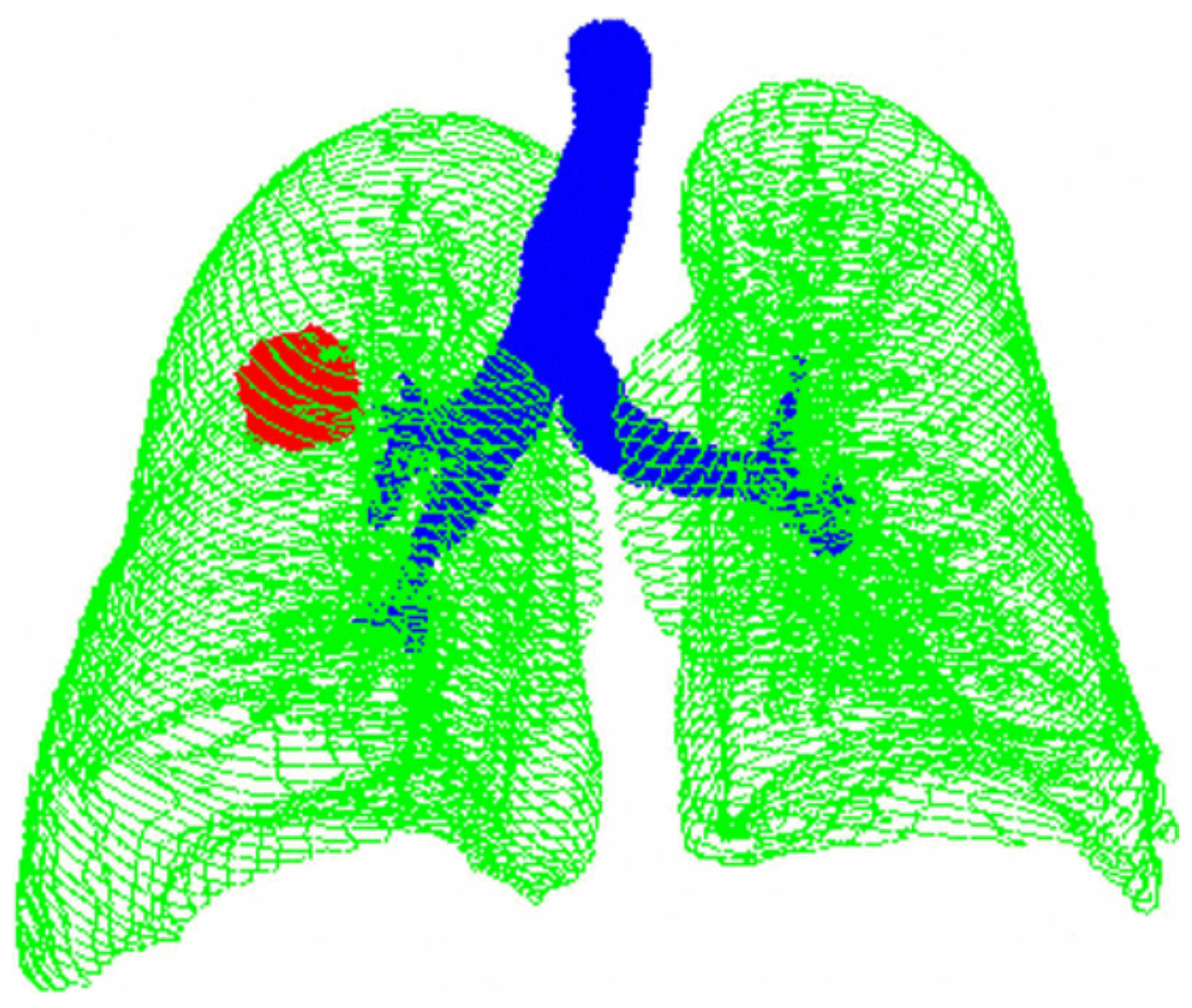

Fig. 3. Illustrates an example of the tumor, lung and trachea segmented volume for one phase of the respiratory cycle.

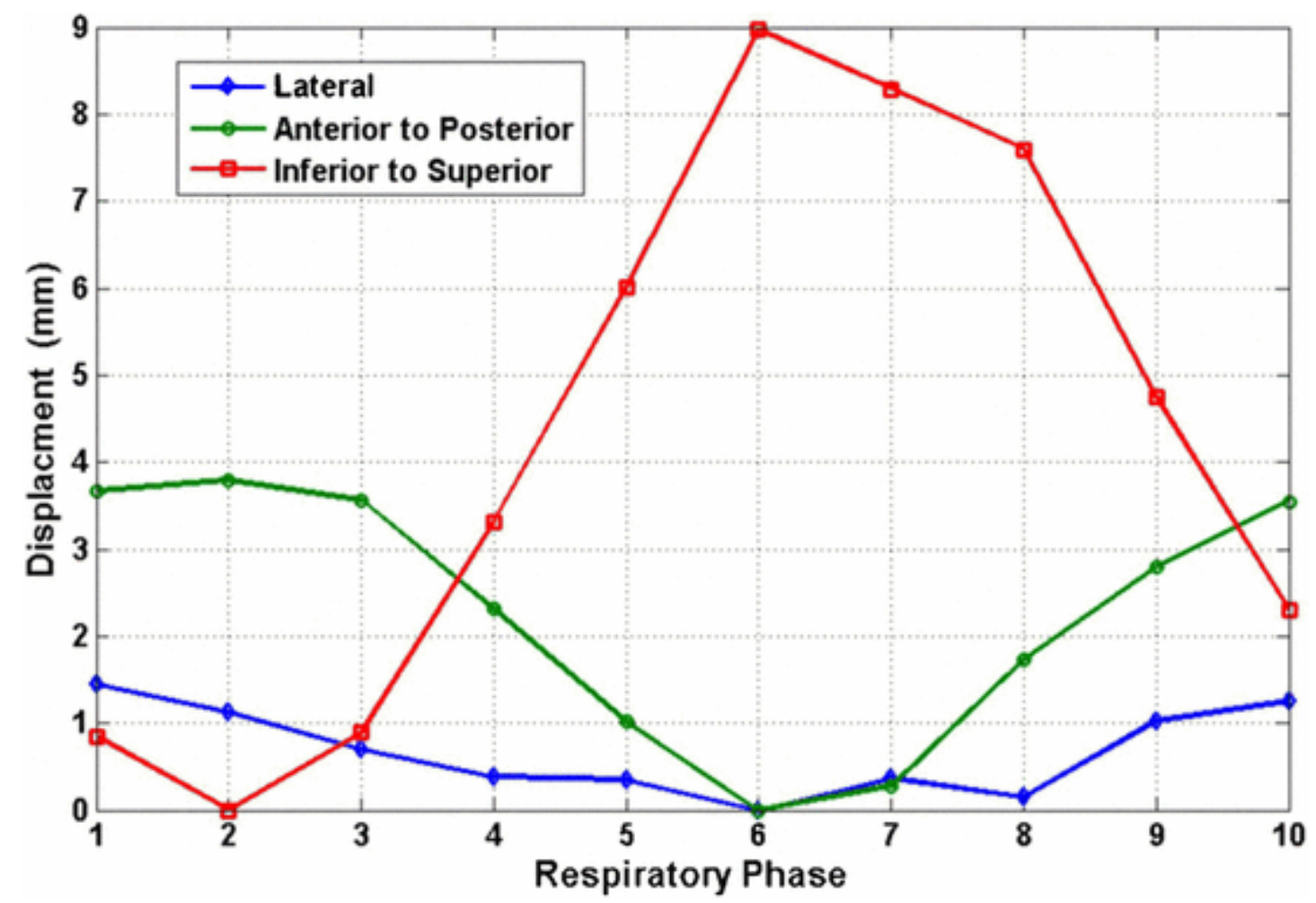

Fig. 4. Illustrates the anterior-posterior (AP) ( $\mathrm{Z}$ direction), lateral (L) ( $\mathrm{X}$ direction), and superiorinferior ( $\mathrm{SI})(Y$ direction) temporal motion of the center gravity of the tumor. 


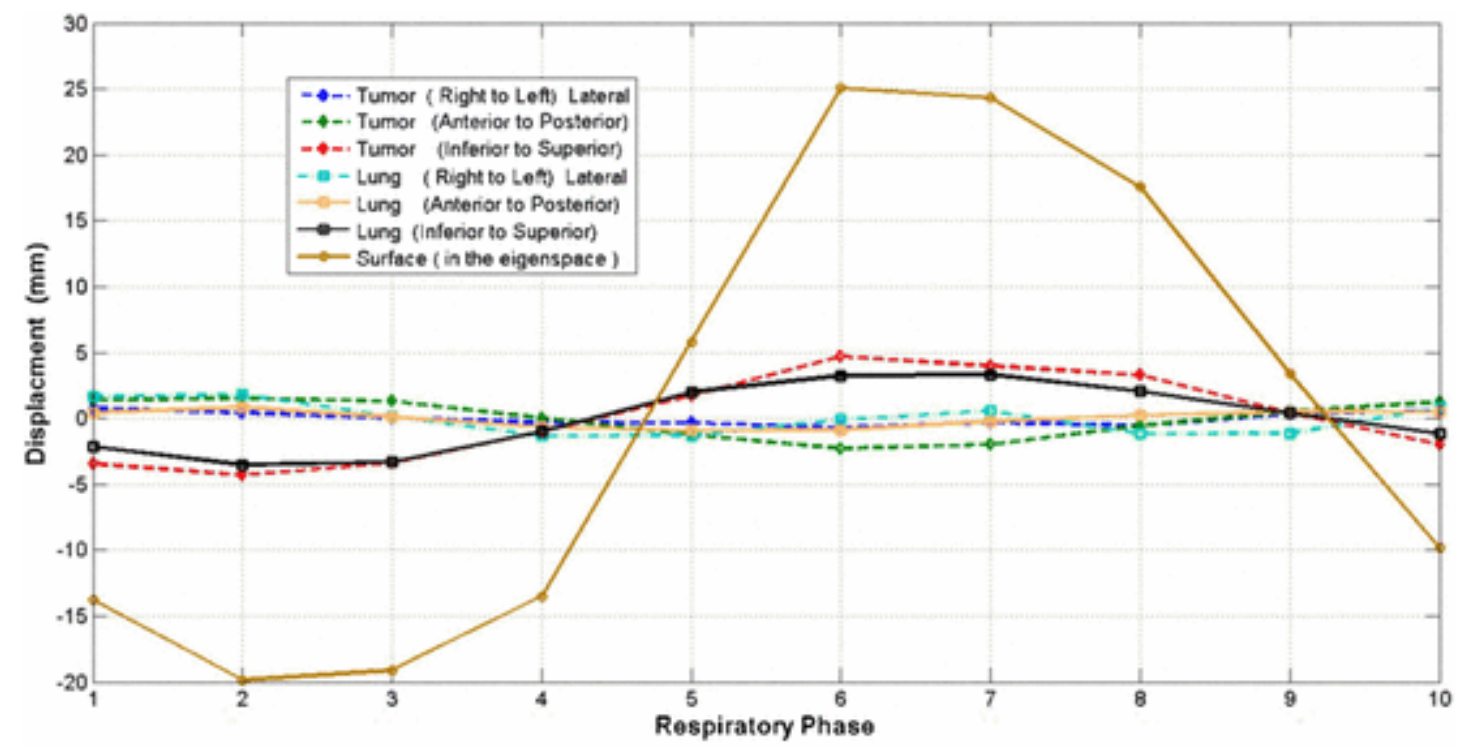

Fig. 5. Illustrates the external surrogate temporal motion in the Eigenspace along with the tumor center of mass anterior-posterior (AP), lateral (L) and superior-inferior (SI) motion.

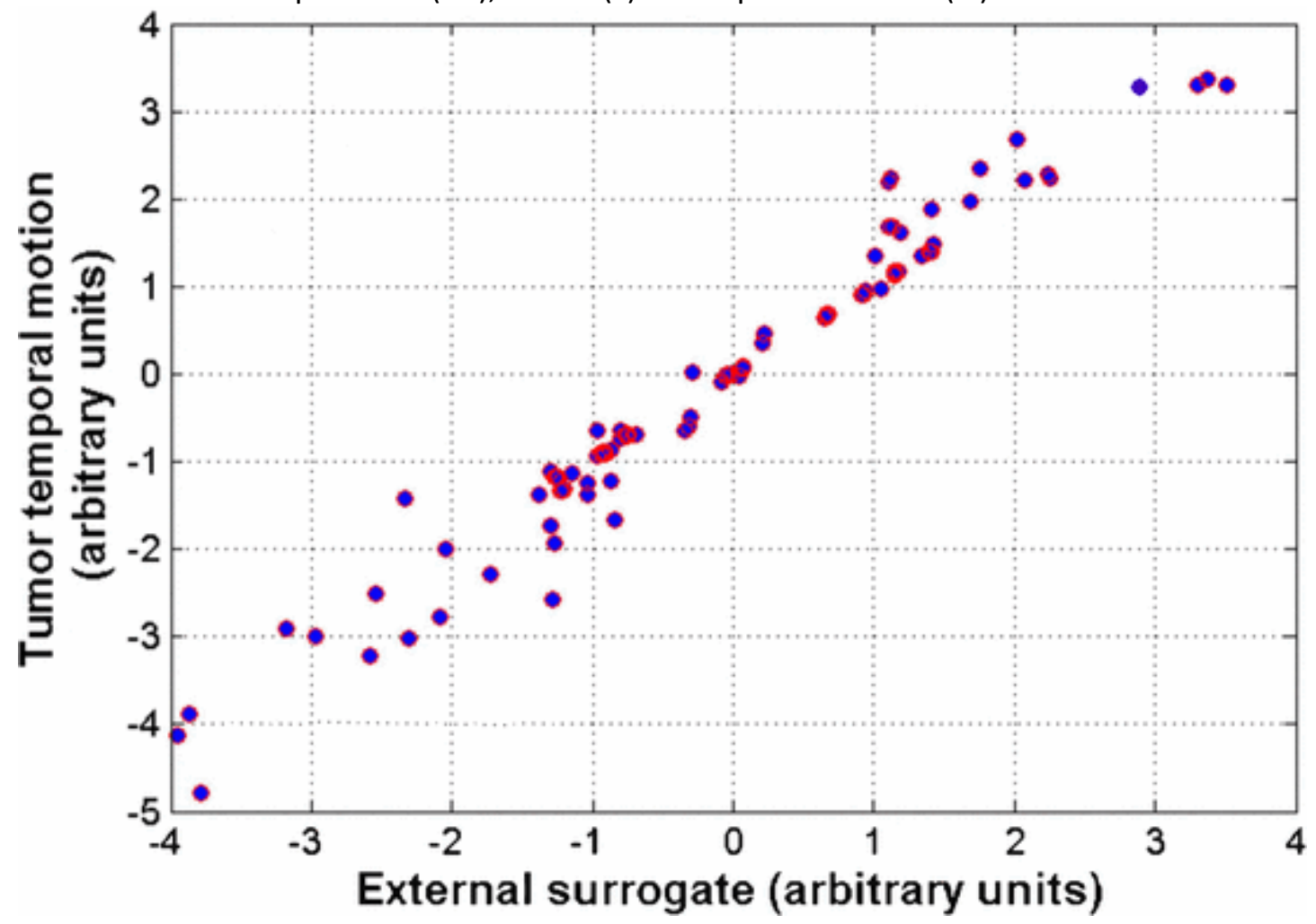

Fig. 6. Illustrates the external surrogate phases verses the tumor phases in the Eigenspace. 


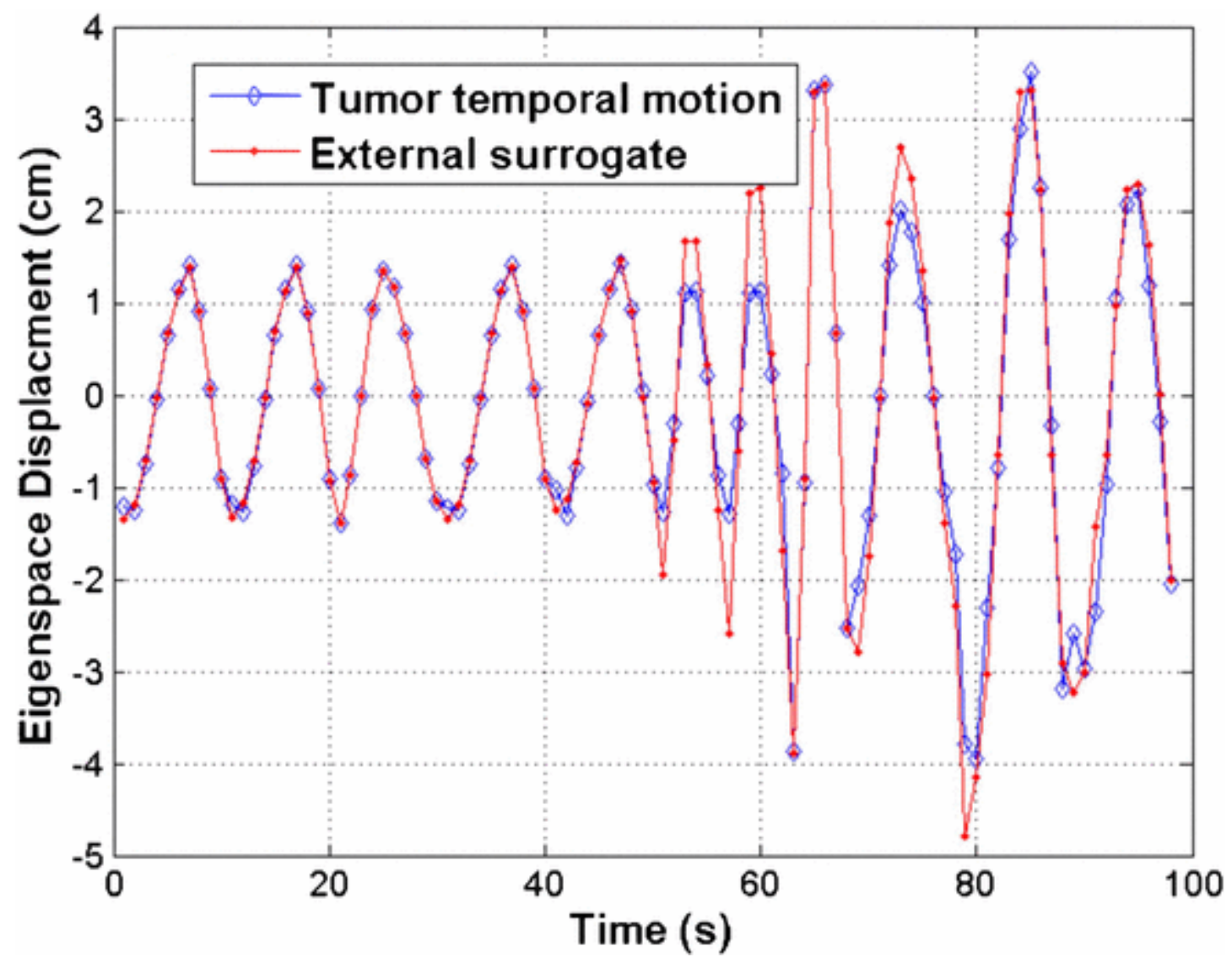

Fig. 7. Illustrates the total data used to train the kernel, where the red line indicates the external surrogate and the blue line indicate the tumor motion in the Eigenspace.

\section{E. The Correlation Prediction Model}

Let $Z$ be a two dimensional vector with a vector elements $w_{P}$ and $w_{C}$ for the external surrogate and tumor motion respectively. The joint probability for each combination $\left(w_{P}, w_{C}\right)$ in $\mathrm{Z}$ is calculated using a Bivariate Kernel Density Estimation. This is accomplished by Equation 3:

$$
P(z)=\frac{1}{n} \sum_{i=0}^{n-1} K_{h}\left(z-Z_{i}\right)
$$

where, $K_{h}(u)$ is a bounded Gaussian kernel function and $z$ a generic vector $\in \Re^{2}$, illustrated in Equation 4:

$$
K_{h}(u)=\frac{1}{h^{2}} K\left(\frac{u}{h}\right)=\frac{1}{\sqrt{2 \pi} h^{2}} e^{\frac{-1}{2}\left(\frac{u}{h}\right)^{2}}
$$

where $\mathbf{h}$ is the Gaussian bandwidth matrix. If we consider that the bandwidth is not the same for each component in $\mathrm{Z}$ and $\mathrm{h}$ is a $2 \times 2$ diagonal matrix with $h_{P}, h_{C}$. Then, Equation 3 can be described by Equation 5: 


$$
\begin{aligned}
P(z) & =P\left(w_{P}, w_{C}\right) \\
& =\frac{1}{n \cdot \operatorname{det}(h)} \sum_{i=0}^{n-1} K\left(\frac{w_{P}-W_{P_{i},}}{h_{w_{P}}}, \frac{w_{C}-W_{C, i}}{h_{w_{C}}}\right)
\end{aligned}
$$

where $K(x, y)=K(x) * K(y)$. The highest likelihood position of the tumor at any external surrogate position $w p$ can be estimated by solving Equation 6 .

$$
\widehat{w_{C}}=\arg g_{\vee\left(w_{C}\right)} \max \left(P\left(w_{P}, w_{C}\right)\right)
$$

Finally, the inverse of the correlation covariance matrix is extracted to estimate the tumor position in the 3D space. For each data set, a leave-one-out method was used to validate the proposed algorithm. The mean distance between the prediction and the actual tumor center of mass was calculated as an error. The average error was about $1.6 \mathrm{~mm}$ in this preliminary analysis.

\section{DISCUSSION AND CONCLUSION}

These preliminary results demonstrate high correlation for all samples, with a correlation coefficient was ranging between 0.87 and 0.99 . This values indicates a high correlation between the external surrogate and tumor respiratory motion. Recasting the internal and external motion into Eigenspace reveals the underlying correlation between surrogate observation and true tumor motion in a compact and optimal manner. producing an average error in tumor position of approximately 1.6 $\mathrm{mm}$.

\section{REFERENCES}

1. M. V. Knopp and H. G. Bischoff Radiologe 34(10), pp. 588-91, 1994.

2. J. Daouk, L. Fin, P. Bailly, and M. E. Meyer Acta Radiol 50(2), pp. 144-55, 2009.

3. H. Shirato, T. Harada, T. Harabayashi, K. Hida, H. Endo, K. Kitamura, R. Onimaru, K. Yamazaki, N. Kurauchi, T. Shimizu, N. Shinohara, M. Matsushita, H. Dosaka-Akita, and K. Miyasaka, "Feasibility of insertion/implantation of 2.0-mm-diameter gold internal fiducial markers for precise setup and realtime tumor tracking in radiotherapy.," International journal of radiation oncology, biology, physics 56, pp. 240-247, May 2003.

4. R. Boutchko, B. W. Reutter, D. Saloner, and G. T. Gullberg, "Correlating motion of internal organs with the displacements of fiducial markers during respiration," in Nuclear Science Symposium Conference Record, 2008. NSS '08. IEEE, pp. 3641-3642, Oct. 2008.

5. M. J. Murphy, "Fiducial-based targeting accuracy for external-beam radiotherapy.," Medical physics 29, pp. 334-344, Mar. 2002.

6. U. Topal and B. Ediz, "Transthoracic needle biopsy: factors effecting risk of pneumothorax.," European journal of radiology 48, pp. 263-267, Dec. 2003.

7. F. Laurent, V. Latrabe, B. Vergier, M. Montaudon, J. M. Vernejoux, and J. Dubrez, "CT-guided transthoracic needle biopsy of pulmonary nodules smaller than $20 \mathrm{~mm}$ : results with an automated 20-gauge coaxial cutting needle.," Clinical radiology 55, pp. 281-287, Apr. 2000. 
8. M. Alnowami, E. Lewis, M. Guy, and K. Wells, "Respiratory motion modelling and prediction using probability density estimation," The 2010 IEEE Nuclear Science Symposium, Medical Imaging Conference, (Knoxville, Tennessee, USA), 2010.

9. M. Alnowani, E. Lewis, M. Guy, and K. Wells, "An observation model for motion correction in nuclear medicine," SPIE, (San Diego, California, USA), 2010.

10. P. A. Yushkevich, J. Piven, H. Cody Hazlett, R. Gimpel Smith, S. Ho, J. C. Gee, and G. Gerig, "Userguided 3D active contour segmentation of anatomical structures: Significantly improved efficiency and reliability," Neuroimage 31(3), pp. 1116-1128, 2006. 